\title{
Early Detection of $A \beta$ Deposition in the 5xFAD Mouse by Amyloid PET
}

\author{
Se Jong Oh, ${ }^{1,2}$ Hae-June Lee, ${ }^{3}$ Kyung Jun Kang $\mathbb{D}^{1},{ }^{1}$ Sang Jin Han, ${ }^{1}$ \\ Yong Jin Lee, ${ }^{1}$ Kyo Chul Lee, ${ }^{1}$ Sang Moo Lim, ${ }^{4}$ Dae Yoon Chi, ${ }^{5}$ Kyeong Min Kim (D), ${ }^{2,6}$ \\ Ji-Ae Park $\left(\mathbb{D},{ }^{1}\right.$ and Jae Yong Choi $\mathbb{D}^{1}$ \\ ${ }^{1}$ Division of RI-Convergence Research, Korea Institute of Radiological and Medical Sciences, Seoul, Republic of Korea
${ }^{2}$ Radiological \& Medico-Oncological Sciences, University of Science and Technology, Daejeon, Republic of Korea
${ }^{3}$ Division of Basic Radiation Bioscience, Korea Institute of Radiological and Medical Sciences, Seoul, Republic of Korea
${ }^{4}$ Department of Nuclear Medicine, Korea Institute of Radiological and Medical Sciences, Seoul, Republic of Korea
${ }^{5}$ Research Institute of Labeling, FutureChem Co., Ltd., Seoul, Republic of Korea
${ }^{6}$ Division of Medical Radiation Equipment, Korea Institute of Radiological and Medical Sciences, Seoul, Republic of Korea
}

Correspondence should be addressed to Ji-Ae Park; jpark@kirams.re.kr and Jae Yong Choi; smhany@kirams.re.kr

Received 18 September 2017; Revised 12 January 2018; Accepted 29 January 2018; Published 28 February 2018

Academic Editor: Anne Roivainen

Copyright (c) 2018 Se Jong Oh et al. This is an open access article distributed under the Creative Commons Attribution License, which permits unrestricted use, distribution, and reproduction in any medium, provided the original work is properly cited.

Purpose. ${ }^{18} \mathrm{~F}$-FC119S is a positron emission tomography (PET) tracer for imaging $\beta$-amyloid (A $\beta$ ) plaques in Alzheimer's disease (AD). The aim of this study is to evaluate the efficacy of ${ }^{18} \mathrm{~F}-\mathrm{FC} 119 \mathrm{~S}$ in quantitating A $\beta$ deposition in a mouse model of early amyloid deposition (5xFAD) by PET. Method. Dynamic ${ }^{18}$ F-FC119S PET images were obtained in 5xFAD $(n=5)$ and wild-type (WT) mice $(n=7)$. The brain PET images were spatially normalized to the M. Mirrione T2-weighted mouse brain MR template, and the volumes of interest were then automatically drawn on the cortex, hippocampus, thalamus, and cerebellum. The specific binding of ${ }^{18} \mathrm{~F}$-FC119S to $\mathrm{A} \beta$ was quantified as the distribution volume ratio using Logan graphical analysis with the cerebellum as a reference tissue. The $\mathrm{A} \beta$ levels in the brain were also confirmed by immunohistochemical analysis. Result. For the 5xFAD group, radioactivity levels in the cortex, the hippocampus, and the thalamus were higher than those for the WT group. In these regions, specific binding was approximately 1.2-fold higher in 5xFAD mice than in WT. Immunohistochemistry supported these findings; the $5 \mathrm{xFAD}$ showed severe $\mathrm{A} \beta$ deposition in the cortex and hippocampus in contrast to the WT group. Conclusion. These results demonstrated that ${ }^{18} \mathrm{~F}$-FC119S PET can successfully distinguish $\mathrm{A} \beta$ depositions in $5 x F A D$ mice from WT.

\section{Introduction}

Alzheimer's disease (AD), the most common neurodegenerative disorder, is characterized by cognitive and memory deterioration as a consequence of abnormal deposition of amyloid-beta $(\mathrm{A} \beta)$ and neurofibrillary tangles of hyperphosphorylated tau [1-3].

Early diagnosis of $\mathrm{AD}$, before the advent of structural changes, is important to reduce the socioeconomic burden of the disease. Molecular imaging with positron emission tomography (PET) is a promising tool because it can functionally detect the burden of $\mathrm{A} \beta$ plaques and evaluate longitudinal changes in patients with $\mathrm{AD}[4,5]$. Radiochemists designed many other $\mathrm{A} \beta$-targeted PET radiotracers in an effort to satisfy one with more drug-like properties. Klunk et al. developed ${ }^{11} \mathrm{C}$-Pittsburgh compound B (PIB), which has high specificity and affinity for $\mathrm{A} \beta$ [6]. However, this radiopharmaceutical has an intrinsic limitation for multicenter studies. ${ }^{11} \mathrm{C}$ has a short half-life of only $20 \mathrm{~min}$, and thus, ${ }^{11} \mathrm{C}$-based radiotracers can be used only at a center with an on-site synthetic facility [7]. Later, ${ }^{18} \mathrm{~F}$-florbetapir, ${ }^{18} \mathrm{~F}$-flutemetamol, and ${ }^{18} \mathrm{~F}$-florbetaben, which detect the presence of $\mathrm{A} \beta$ plaques, were developed and approved by the US Food and Drug Administration [8-10]. Previous PET studies have shown that they have both succeeded and failed in detecting amyloidosis in AD animal models [11-15]. 
Recently, ${ }^{18}$ F-FC119S (2-[2-(N-monomethyl)aminopyridine6-yl]-6-[(S)-3-fluoro-2-hydroxypropoxy]benzothazole) is developed $\mathrm{A} \beta$ imaging PET tracer. This tracer has high selectivity and metabolic stability against troublesome in vivo defluorination [16]. In addition, ${ }^{18} \mathrm{~F}$-FC119S showed a greater binding value in the APP/PS1 than their wild-type (WT) counterparts [17]. In the first in human study including patients with mild cognitive impairment and AD, ${ }^{18} \mathrm{~F}-\mathrm{FC} 119 \mathrm{~S}$ displays significant linear correlation with ${ }^{11}$ C-PIB [18].

Several transgenic mouse lines have been used for amyloid PET research, but only a limited range of $\mathrm{AD}$ mice can be used $[14,15]$. The $5 x F A D$ mouse is a transgenic model of AD carrying five mutations associated with early onset familial Alzheimer's disease (FAD): the K670N/M671L (Swedish), I716V (Florida), and V717I (London) mutations in human amyloid precursor protein (APP) and the M146L and L286V mutations in human presenilin-1 (PS1) [19-22]. All five FAD mutations synergistically promote the occurrence of $\mathrm{A} \beta_{42}$; therefore, this model shows very aggressive $\mathrm{A} \beta$ deposition in the brain starting at 1.5 months of age [23].

The purpose of the present study is to examine the efficacy of ${ }^{18} \mathrm{~F}$-FC119S PET for distinguishing $\mathrm{A} \beta$ deposition in an $\mathrm{AD}$ mouse model of early amyloid deposition from WT.

\section{Materials and Methods}

2.1. Animals. Two groups of mice were used for these studies: $5 x F A D$ mice (male, $n=5$ ) and WT counterparts (male, $n=7$ ), all at the age of 5.5 months. The care, maintenance, and treatment of animals in these studies followed protocols approved by the Institutional Animal Care and Use Committee of Korea Institute of Radiological \& Medical Sciences (KIRAMS), and the experiments involving animals were performed according to the Guide for the Care and Use of Laboratory Animals published by the US National Institutes of Health. The animal housing room was automatically controlled with a temperature of $22 \pm 3^{\circ} \mathrm{C}$, a $12 \mathrm{~h} / 12 \mathrm{~h}$ light/dark cycle and $55 \pm 20 \%$ humidity. Sterilized rodent diet and purified tap water were supplied ad libitum.

2.2. Preparation of ${ }^{18}$ F-FC119S. ${ }^{18}$ F-FC119S was synthesized by nucleophilic substitution of F-18 on the precursor as previously described in Byun et al's work [18]. The specific activity of ${ }^{18} \mathrm{~F}$-FC119S was greater than $44 \mathrm{GBq} / \mu \mathrm{mol}$, and the mean radiochemical purity was $99 \%(n=4)$.

2.3. PET/CT Scan. PET images of the mice were obtained using a small-animal PET scanner (nanoScan ${ }^{\circledR}$, Mediso, Budapest, Hungary), consisting of two rings with $12 \mathrm{PET}$ detector modules. The spatial resolution at $1 \mathrm{~mm}$ off the center of the scanner in the axial, radial, tangential directions is $0.85,0.8$, and $0.8 \mathrm{~mm}$, respectively. Mice were anesthetized with $2.5 \%$ isoflurane, and ${ }^{18} \mathrm{~F}-\mathrm{FC} 119 \mathrm{~S}(8.9 \pm 1.3 \mathrm{MBq} / 200 \mathrm{uL})$ was injected through the tail vein with a syringe pump (KDS 210, KD Scientific, Holliston, MA) over the course of $1 \mathrm{~min}$. Simultaneously, dynamic PET scanning was performed for $60 \mathrm{~min}$. Images were reconstructed with user defined time frames $(14 \times 30$ s, $3 \times 60$ s, $4 \times 300$ s, $3 \times 600$ s, 24 frames in total) using a 3-dimensional ordered subset expectation maximization algorithm. For attenuation correction and anatomical reference, a computed tomography (CT) scan was acquired immediately after PET $(50 \mathrm{kVp}$ of X-ray voltage at $0.16 \mathrm{mAs})$.

2.4. Image Analysis. According to Oakley et al., A $\beta$ deposition begins to appear in the cortex, the hippocampus and the thalamus of $5 x F A D$ mice at 1.5 months of age [23]. However, no significant increase in $A \beta$ was found in the olfactory bulbs, striatum, hypothalamus, or thalamus at 5 months of age [24]. Therefore, we selected the cortex, hippocampus, and the thalamus as volumes of interest (VOIs). To create a study-specific PET brain template, dynamic PET images were motion-corrected and spatially normalized to the T2-weighted mouse brain MR template (M. Mirrione, embedded PMOD software). Four VOIs were defined on the MRI template; cortex $\left(149.2 \mathrm{~mm}^{3}\right)$, hippocampus $\left(24.9 \mathrm{~mm}^{3}\right)$, thalamus $\left(28.2 \mathrm{~mm}^{3}\right)$, and cerebellum $\left(57.2 \mathrm{~mm}^{3}\right.$, Figure 1). Finally, regional time-activity curves (TACs) were generated. The obtained uptake value was determined for each brain region and presented as a standardized uptake value (SUV). The SUVs were obtained by normalizing tissue radioactivity concentration to injected dose and body weight [25].

To compare the specific binding of ${ }^{18} \mathrm{~F}$-FC119S to $\mathrm{A} \beta$ deposits, we used Logan graphical analysis $\left(t^{*}=10 \mathrm{~min}\right)$ to derive a distribution volume ratio (DVR) based on the cerebellum [26]. All image analysis was conducted with PMOD (version 3.4, PMOD Group, Graubunden, Switzerland).

2.5. Immunohistochemistry. The animals were euthanized, and brain samples were fixed in $4 \%$ paraformaldehyde for 48 hours, embedded in paraffin, and sectioned at $5 \mu \mathrm{m}$ intervals. Immunohistochemistry was conducted using the Vectastain Elite ABC kit (Vector Laboratories Inc., Burlingame, CA, USA) following the manufacturer's protocol. For antigen retrieval, the sections were placed in a citrate buffer $(\mathrm{pH}$ 6.0) and heated in boiling water for 30 minutes. The sections were then placed in $0.3 \% \mathrm{H}_{2} \mathrm{O}_{2}$ in absolute methanol for 15 minutes at room temperature to block the endogenous peroxidase. Sections were then incubated overnight at $4^{\circ} \mathrm{C}$ with mouse anti-6E10 antibody $(1: 1000$, SIG-39320, Covance, Emeryville, CA), washed, and incubated with the corresponding secondary antibody. Image J was used to quantify the amount of $\mathrm{A} \beta$ in each brain section. As a control, the primary antibody was omitted from several test sections in each experiment. The sections were counterstained with Harris' hematoxylin prior to mounting.

For analysis of the amyloid plaques, the ImageJ software (https://rsbweb.nih.gov/ij/) was used. After adjusting for threshold, Image J was used to measure total area of the plaques and the percentage of total brain area occupied by plaques. The brain area (cortex or hippocampus) was outlined in the right hemisphere using the edit plane function. Data were pooled from $8 \sim 12$ sections at $\times 200$ magnification of each mouse and 5 mice were used for the statistical analysis.

2.6. Statistical Analysis. The quantitative results are expressed as the mean $\pm \mathrm{SD}$. All statistical results were analyzed 


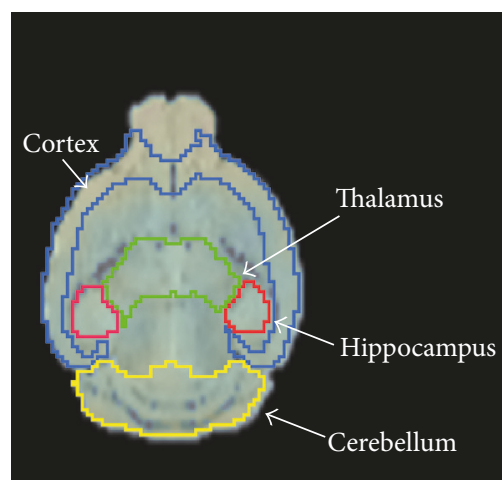

(a)

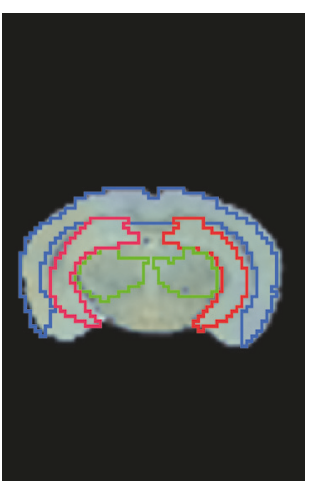

(b)

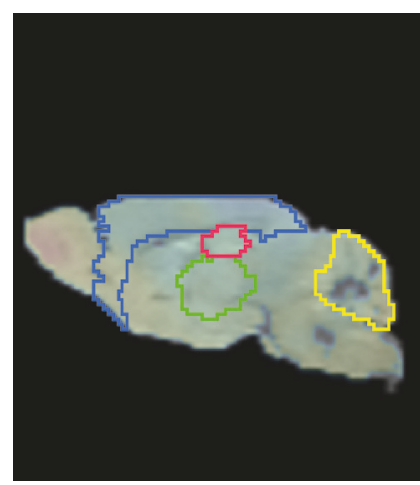

(c)

FIGURE 1: Definition of VOIs from a 5xFAD mouse in the horizontal (a), coronal (b), and sagittal (c) planes. The PET was spatially normalized to the T2-weighted mouse brain MR template.

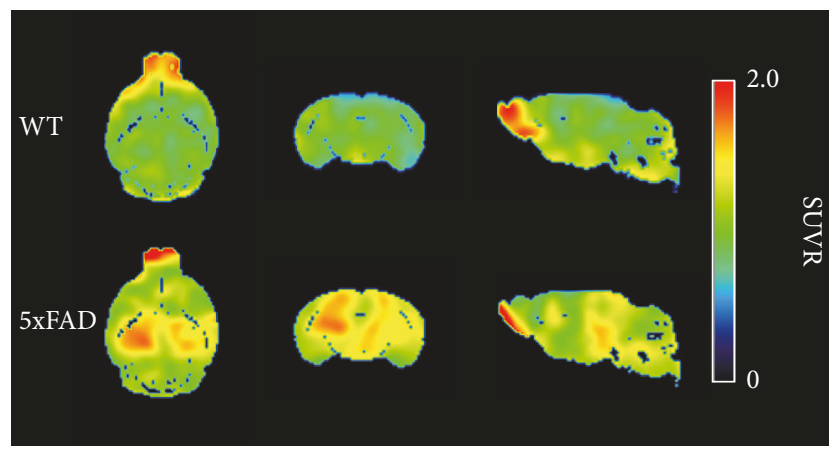

Figure 2: ${ }^{18}$ F-FC119S summed PET images (30-60 min) of the WT and the $5 x F A D$ in the one representative animal. The color scale indicates the SUV ratio.

with Prism (GraphPad Software, Inc., CA). Student's $t$-test was used to determine statistical significance at the $95 \%$ confidence level, with $p<0.05$ indicating a significant difference.

\section{Results}

3.1. ${ }^{18}$ F-FC119S PET Images. The ability of ${ }^{18} \mathrm{~F}-\mathrm{FC} 119 \mathrm{~S}$ to quantify $\mathrm{A} \beta$ burden was assessed by PET. PET images showing the brain SUV ratio (30-60 min) are shown in Figure 2. By visual inspection, the $5 x F A D$ showed higher cortical and hippocampal ${ }^{18}$ F-FC119S uptake than the WT group. Radioactivity was also detected in the olfactory bulb in both groups, but this uptake was due to spill-over from the Harderian gland [27].

Figure 3 represents the regional TACs. After approximately 10 minutes, the cortex and hippocampus and the thalamus of the $5 \mathrm{xFAD}$ showed greater uptake than those of the WT (Figures 3(a)-3(c)). In contrast, radioactivities in the cerebellum, which we used as the reference region, did not differ between groups (Figure 3(d)). Area under the curve (AUC) values was obtained from 30 to 60 minutes by the trapezoid rule (Figures $3(\mathrm{e})-3(\mathrm{~h})$ ). In 5xFAD mice, the AUC values of the cortex, hippocampus, and thalamus showed
$33.3,41.7$, and $25.9 \%$ increments, respectively, compared with the corresponding values in WT mice. Among VOIs, only hippocampus showed statistically significant difference $(p=$ 0.0479 ). The difference of cerebellar uptakes between $5 x F A D$ and WT was not statistically significant $(p=0.9299)$.

3.2. Distribution Volume Ratios. To elucidate the specific binding level of ${ }^{18}$ F-FC119S, we calculated the DVR values (Figure 4). The mean DVR values for the $5 x$ FAD were $10-21 \%$ higher than those for the WT group (DVR values in cortex: $5 \mathrm{xFAD}=0.99 \pm 0.05$ versus $\mathrm{WT}=0.86 \pm 0.04, p=0.0007$; in hippocampus: $5 \mathrm{xFAD}=1.03 \pm 0.09$ versus $\mathrm{WT}=0.85 \pm 0.03$, $p=0.0006$; in thalamus: $5 \times \mathrm{XAD}=1.01 \pm 0.12$ versus $\mathrm{WT}=$ $0.92 \pm 0.04, p=0.0748$ ).

3.3. Immunohistochemical Staining. To assess the actual $\mathrm{A} \beta$ burden in the brain, we performed immunohistochemistry (Figure 5). While $\mathrm{A} \beta$ deposition was not detected in the WT mice, increased $\mathrm{A} \beta$ burden was identified in $5 \mathrm{xFAD}$ (Figure 5(a)). The quantification revealed that the $A \beta$ deposition was greater in the cortex than in the hippocampus (Figure S1, $6.13 \pm 0.76 \%$ and $3.08 \pm 0.73 \%$, resp.). These results were consistent with previous studies showing that $\mathrm{A} \beta$ filled most of the hippocampus and cortex of $5 \mathrm{xFAD}$ mice within 6 months [28]. However, A $\beta$ plaques were not expressed in both $5 x F A D$ and WT (Figure S2). The association between $\mathrm{A} \beta$ deposition and DVRs showed that the cortex has low correlation (Figure 5(b), $r=0.45$ ) whereas the hippocampus has strong correlation (Figure 5(c), $r=0.85$ ).

\section{Discussion}

To the best of our knowledge, the present study is the first to demonstrate that ${ }^{18}$ F-FC119S PET can successfully differentiate the $\mathrm{A} \beta$ burden of $5 \mathrm{xFAD}$ mice from that of WT mice. These PET results were validated by immunohistochemistry. From this perspective, ${ }^{18} \mathrm{~F}-\mathrm{FC} 119 \mathrm{~S}$ may be a useful PET radiotracer for $A \beta$ imaging.

Pathologically, $\mathrm{A} \beta$ deposition in the brain occurs first in the cortical area (6-9 months), followed by the hippocampus (12-15 months) [29-32]. This implies that $\mathrm{A} \beta$ plaques have 

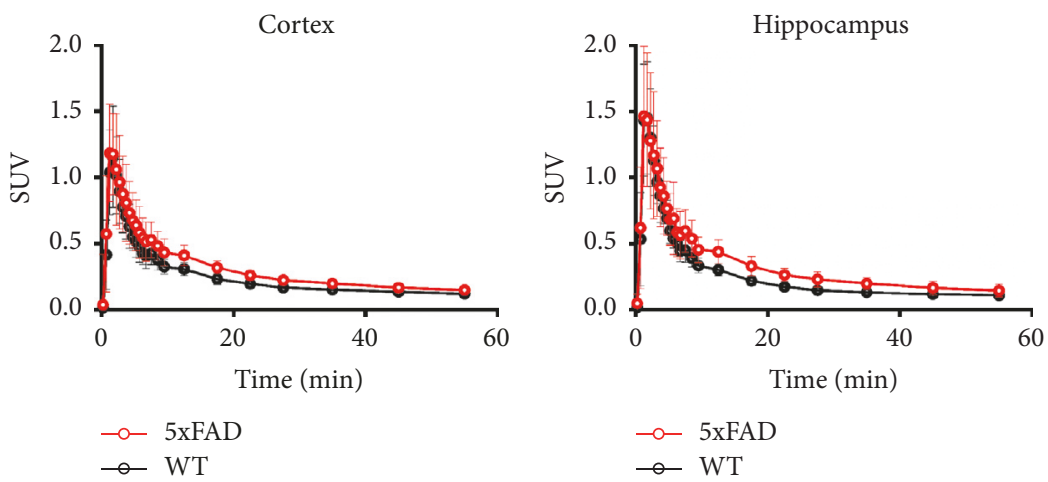

(a)

(b)
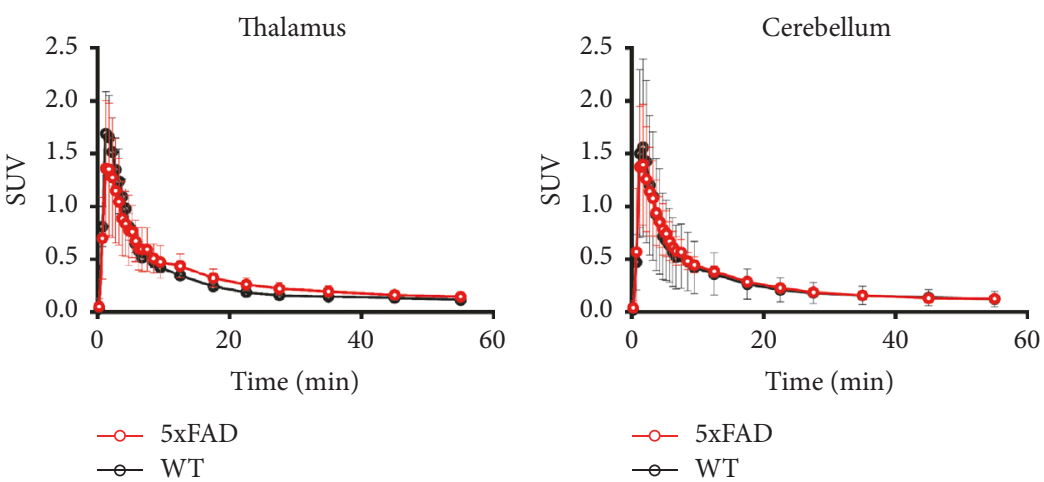

(c)

(d)
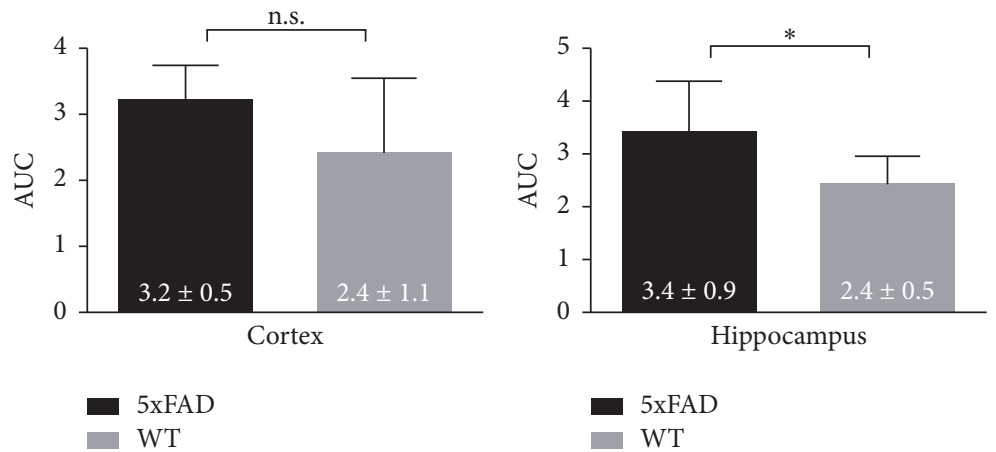

(e)

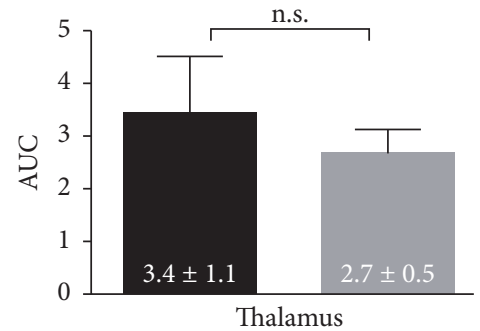

(f)

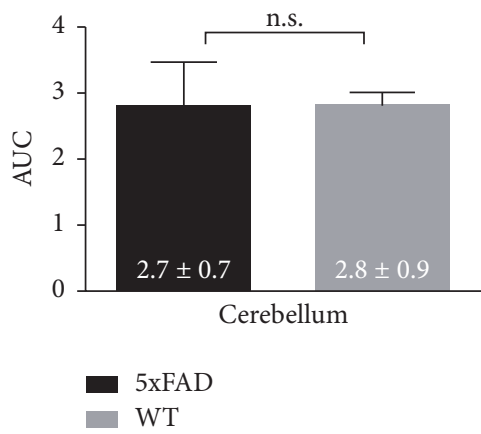

(h)

Figure 3: Time-activity curves of the cortex (a), hippocampus (b), thalamus (c), and cerebellum (d). Area under the curve (AUC) for the cortex (e), hippocampus (f), thalamus (g), and cerebellum (h). Data are presented as the mean \pm SD. $\left({ }^{*} p<0.05\right.$, n.s. $=$ statistically nonsignificant difference). 


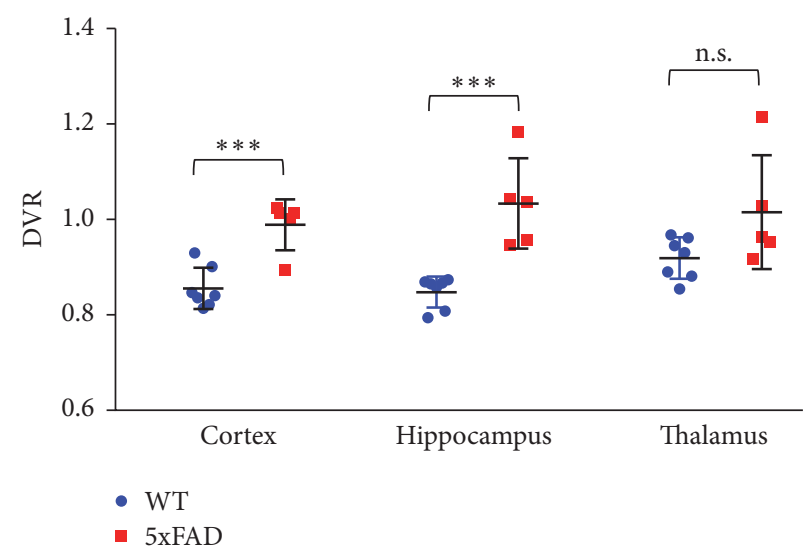

FIgURE 4: The DVR values according to Logan graphical analysis. Statistical significance was defined as a $p$ value less than $0.05{ }^{* * *} p<0.001$, n.s. = statistically nonsignificant difference).

WT
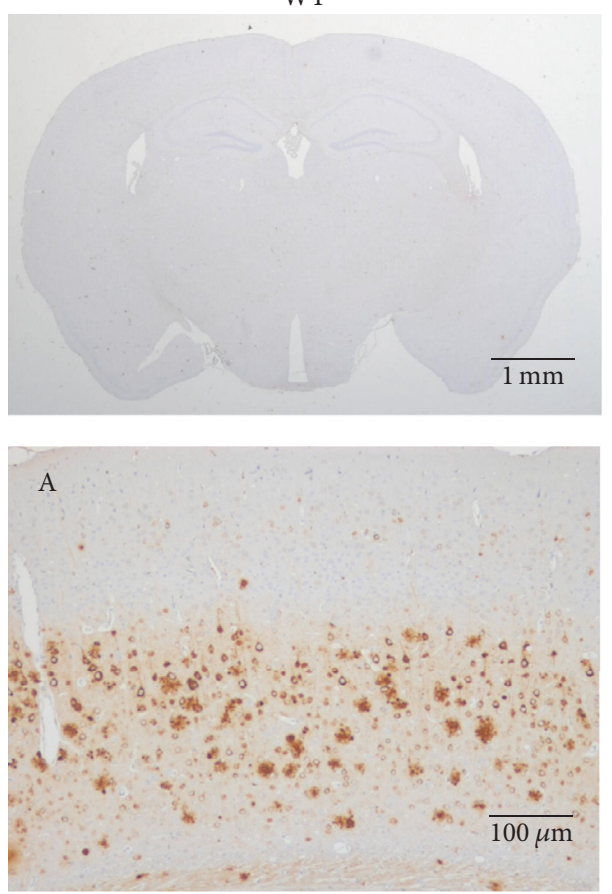

(a)

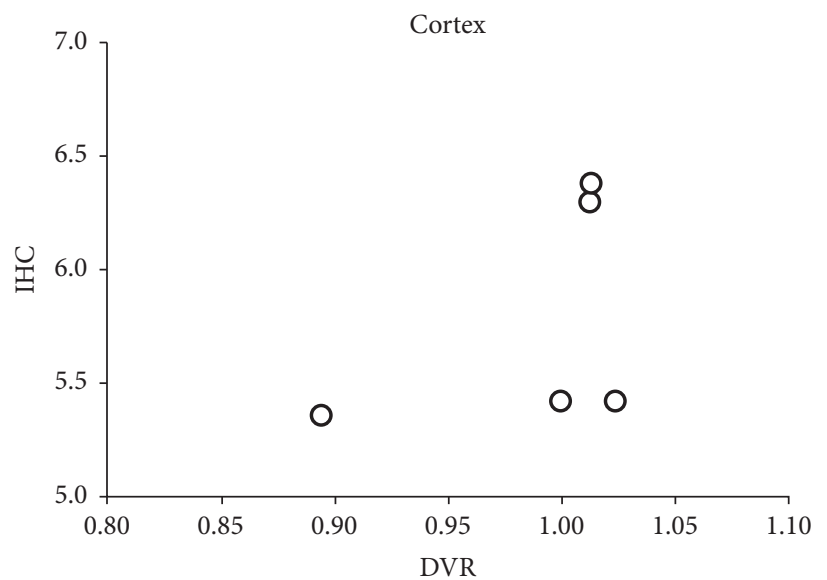

(b)
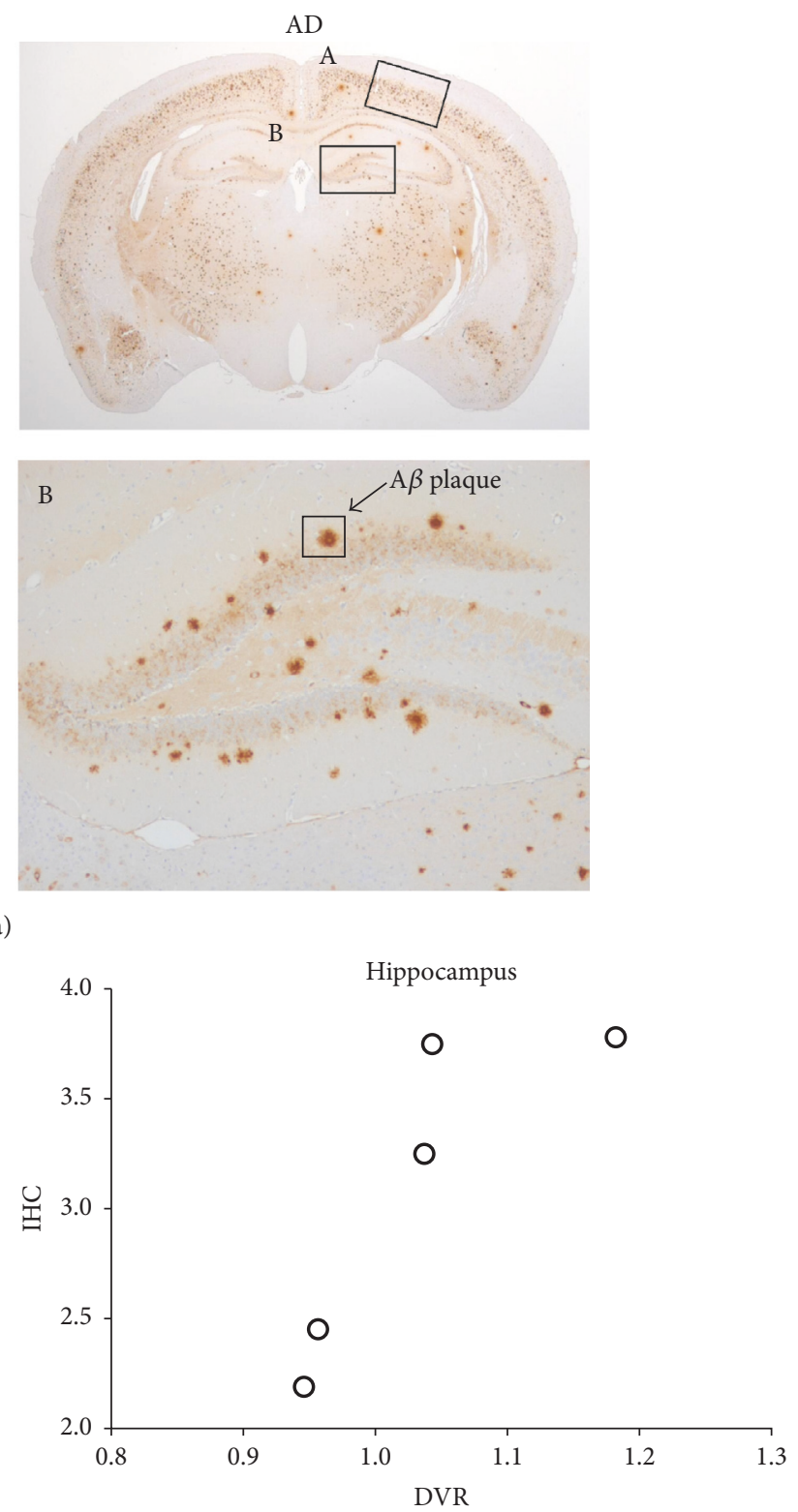

(c)

FIGURE 5: Immunohistochemical staining of $\mathrm{A} \beta$ in the brains of WT and 5xFAD mice (a). Insets represent high-magnification images of the cortex (A) and hippocampus (B). Relationship between IHC data and DVRs of cortex (b) and hippocampus (c). 
a relatively lower concentration in the limbic system than in the cortex at a young age [30]. Previous rodent amyloid PET studies using ${ }^{11} \mathrm{C}$-PIB or ${ }^{18} \mathrm{~F}$-florbetaben failed to discriminate between young AD mice (PS2APP, G384A, APP/PS1; all mice age less than 12 months) and WT mice in terms of specific binding in the hippocampus [15], whereas the differences of binding values in 20-month-aged APP/PS1 mice were successfully detected by ${ }^{18}$ F-FC119S [17]. Rojas et al. also demonstrated that increased cerebral uptakes in 10-16month-aged $5 x F A D$ by ${ }^{18} \mathrm{~F}$-florbetapir [12]. In addition to genetic modification, age may be a critical factor. Pathological changes in the hippocampus are observable in advanced $\mathrm{AD}$ mice. Longitudinal PET studies must be conducted for 20 months or longer to detect these changes [33]. However, this long timeframe makes it difficult to choose an appropriate age for analysis and to maintain the animals in good condition for the entire study. 5xFAD line displays high concentration of $\mathrm{A} \beta$ as well as cognitive dysfunction around 4 months of age. Moreover, these mice develop neuron loss, unlike most other hAPP and hAPP/PS1 models [34]. Regarding this issue, $5 x F A D$ mice may offer an advantage over other transgenic mice. Due to the aggressive $\mathrm{A} \beta$ deposition in this model, we confirmed the presence of detectable changes even at the age of 5.5 months. The benefit of $5 x F A D$ model is the early amyloid deposition, which could offer to longitudinal experiments over APP/PS1.

The current PET results are an underestimate of the in vitro values. In our immunohistochemistry analysis, the cortical and hippocampal $\mathrm{A} \beta$ plaque concentration was evidently increased in the $5 x F A D$, while no $A \beta$ was detected in WT mice. Despite this in vitro result, the difference between binding values was only $16-22 \%$. These patterns are consistent with previous studies using APP/PS1 transgenic mice $[17,19]$. This observation may be related to experimental techniques. Dynamic PET images provide information on the pharmacokinetics and pharmacodynamics of the tracer in the body [35]. On the other hand, histological examination reflects antigen-antibody binding ability and is observed in a brain slice under static incubation conditions. Partial volume effect by the small brain structure and limited PET resolution also attribute the underestimation of PET signal.

\section{Conclusion}

In summary, ${ }^{18} \mathrm{~F}-\mathrm{FC} 119 \mathrm{~S}$ PET showed selective binding to pathological $\mathrm{A} \beta$ deposits in 5xFAD mice. The cortical and hippocampal uptake of the tracer was higher in the $5 \mathrm{xFAD}$ than in WT mice. These results were reproduced by immunohistochemistry. Therefore, ${ }^{18}$ F-FC119S would be a suitable candidate preclinical PET radiotracer.

\section{Conflicts of Interest}

The authors declare that they have no conflicts of interest.

\section{Acknowledgments}

FutureChem (Korea) is acknowledged for the synthesis of ${ }^{18}$ F-FC119S. This study was supported by a grant from the Korea Institute of Radiological and Medical Sciences (KIRAMS), which is funded by the Ministry of Science and ICT (MSIT), Republic of Korea (1711062441/50461-2018, 50536-2018).

\section{Supplementary Materials}

Figure S1: quantification of $\mathrm{A} \beta$ deposition in the cortex and hippocampus. Values are presented as the mean \pm SD; n.d.: not detected. Figure S2: immunohistochemical staining of $\mathrm{A} \beta$ in the cerebellum of $\mathrm{WT}$ and $5 \mathrm{xFAD}$ mice. (Supplementary Materials)

\section{References}

[1] G. Poisnel, A.-S. Hérard, N. El Tannir El Tayara et al., "Increased regional cerebral glucose uptake in an APP/PS1 model of Alzheimer's disease," Neurobiology of Aging, vol. 33, no. 9, pp. 1995-2005, 2012.

[2] V. L. Villemagne, K. E. Pike, D. Darby et al., "A $\beta$ deposits in older non-demented individuals with cognitive decline are indicative of preclinical Alzheimer's disease," Neuropsychologia, vol. 46, no. 6, pp. 1688-1697, 2008.

[3] D. J. Selkoe, "Alzheimer's disease: genes, proteins, and therapy," Physiological Reviews, vol. 81, no. 2, pp. 741-766, 2001.

[4] A. Nordberg, "PET imaging of amyloid in Alzheimer's disease," The Lancet Neurology, vol. 3, no. 9, pp. 519-527, 2004.

[5] K. A. Johnson, N. C. Fox, R. A. Sperling, and W. E. Klunk, "Brain imaging in Alzheimer disease," Cold Spring Harbor Perspectives in Medicine, vol. 2, no. 4, 2012.

[6] W. E. Klunk, H. Engler, A. Nordberg et al., "Imaging brain amyloid in Alzheimer's disease with Pittsburgh Compound-B," Annals of Neurology, vol. 55, no. 3, pp. 306-319, 2004.

[7] J. M. Mountz, C. M. Laymon, A. D. Cohen et al., "Comparison of qualitative and quantitative imaging characteristics of $\left[{ }^{11} \mathrm{C}\right] \mathrm{PiB}$ and $\left[{ }^{18} \mathrm{~F}\right]$ flutemetamol in normal control and Alzheimer's subjects," NeuroImage: Clinical, vol. 9, pp. 592-598, 2015.

[8] I.-T. Hsiao, C.-C. Huang, C.-J. Hsieh et al., "Correlation of early-phase 18F-florbetapir (AV-45/Amyvid) PET images to FDG images: Preliminary studies," European Journal of Nuclear Medicine and Molecular Imaging, vol. 39, no. 4, pp. 613-620, 2012.

[9] N. Nelissen, K. Van Laere, L. Thurfjell et al., "Phase 1 study of the Pittsburgh compound $\mathrm{B}$ derivative ${ }^{18} \mathrm{~F}$-flutemetamol in healthy volunteers and patients with probable Alzheimer disease," Journal of Nuclear Medicine, vol. 50, no. 8, pp. 1251$1259,2009$.

[10] G. J. O'Keefe, T. H. Saunder, S. Ng et al., "Radiation dosimetry of $\beta$-amyloid tracers ${ }^{11} \mathrm{C}-\mathrm{PiB}$ and ${ }^{18} \mathrm{~F}$-BAY94-9172," Journal of Nuclear Medicine, vol. 50, no. 2, pp. 309-315, 2009.

[11] G. Poisnel, M. Dhilly, O. Moustié et al., "PET imaging with $\left[{ }^{18} \mathrm{~F}\right] \mathrm{AV}-45$ in an APP/PS1-21 murine model of amyloid plaque deposition," Neurobiology of Aging, vol. 33, no. 11, pp. 2561-2571, 2012.

[12] S. Rojas, J. R. Herance, J. D. Gispert et al., "In vivo evaluation of amyloid deposition and brain glucose metabolism of 5XFAD mice using positron emission tomography," Neurobiology of Aging, vol. 34, no. 7, pp. 1790-1798, 2013.

[13] B. Von Reutern, B. Grünecker, B. H. Yousefi, G. Henriksen, M. Czisch, and A. Drzezga, "Voxel-based analysis of amyloidburden measured with $\left[{ }^{11} \mathrm{c}\right]$ pib pet in a double transgenic 
mouse model of alzheimer's disease," Molecular Imaging and Biology, vol. 15, no. 5, pp. 576-584, 2013.

[14] A. Snellman, F. R. López-Picón, J. Rokka et al., "Longitudinal amyloid imaging in mouse brain with 11C-PIB: Comparison of APP23, Tg2576, and APPswe-PS1dE9 mouse models of Alzheimer disease," Journal of Nuclear Medicine, vol. 54, no. 8, pp. 1434-1441, 2013.

[15] M. Brendel, A. Jaworska, E. Grießinger et al., "Cross-sectional comparison of small animal $\left[{ }^{18} \mathrm{~F}\right]$-florbetaben amyloid-PET between transgenic AD mouse models," PLOS ONE, vol. 10, no. 2, Article ID e0116678, 2015.

[16] B. S. Lee, S. Y. Chu, H. R. Kwon et al., "Synthesis and evaluation of 6-(3-[ $\left.{ }^{18} \mathrm{~F}\right]$ fluoro-2-hydroxypropyl)-substituted 2-pyridylbenzothiophenes and 2-pyridylbenzothiazoles as potential PET tracers for imaging A $\beta$ plaques," Bioorganic \& Medicinal Chemistry, vol. 24, no. 9, pp. 2043-2052, 2016.

[17] S. J. Oh, M. H. Kim, S. J. Han et al., "Preliminary PET Study of ${ }^{18}$ F-FC119S in Normal and Alzheimer's Disease Models," Molecular Pharmaceutics, vol. 14, no. 9, pp. 3114-3120, 2017.

[18] B. H. Byun, B. I. Kim, S. Y. Park et al., "Head-to-head comparison of ${ }^{11} \mathrm{C}-\mathrm{PiB}$ and ${ }^{18} \mathrm{~F}-\mathrm{FC} 119 \mathrm{~S}$ for $\mathrm{A} \beta$ imaging in healthy subjects, mild cognitive impairment patients, and Alzheimer's disease patients," Medicine (United States), vol. 96, no. 12, Article ID e6441, 2017.

[19] M. Mullan, F. Crawford, K. Axelman et al., "A pathogenic mutation for probable Alzheimer's disease in the APP gene at the N-terminus of $\beta$-amyloid," Nature Genetics, vol. 1, no. 5, pp. 345-347, 1992.

[20] C. B. Eckman, N. D. Mehta, R. Crook et al., "A new pathogenic mutation in the APP gene (1716V) increases the relative proportion of A $\beta 42(43)$," Human Molecular Genetics, vol. 6, no. 12, pp. 2087-2089, 1997.

[21] A. Goate, M.-C. Chartier-Harlin, M. Mullan et al., "Segregation of a missense mutation in the amyloid precursor protein gene with familial Alzheimer's disease," Nature, vol. 349, no. 6311, pp. 704-706, 1991.

[22] M. Citron, C. B. Eckman, T. S. Diehl et al., "Additive effects of PS1 and APP mutations on secretion of the 42- residue amyloid $\beta$-protein," Neurobiology of Disease, vol. 5, no. 2, pp. 107-116, 1998.

[23] H. Oakley, S. L. Cole, S. Logan et al., "Intraneuronal $\beta$-amyloid aggregates, neurodegeneration, and neuron loss in transgenic mice with five familial Alzheimer's disease mutations: potential factors in amyloid plaque formation," The Journal of Neuroscience, vol. 26, no. 40, pp. 10129-10140, 2006.

[24] I. R. Macdonald, D. R. DeBay, G. A. Reid et al., "Early detection of cerebral glucose uptake changes in the 5XFAD mouse," Current Alzheimer Research, vol. 11, no. 5, pp. 450-460, 2014.

[25] C. G. Schwarz, M. L. Senjem, J. L. Gunter et al., "Optimizing PiB-PET SUVR change-over-time measurement by a largescale analysis of longitudinal reliability, plausibility, separability, and correlation with MMSE," NeuroImage, vol. 144, pp. 113-127, 2017.

[26] J. Logan, J. S. Fowler, N. D. Volkow, G.-J. Wang, Y.-S. Ding, and D. L. Alexoff, "Distribution volume ratios without blood sampling from graphical analysis of PET data," Journal of Cerebral Blood Flow \& Metabolism, vol. 16, no. 5, pp. 834-840, 1996.

[27] K. Matsubara, M. Ibaraki, H. Shimada et al., "Impact of spillover from white matter by partial volume effect on quantification of amyloid deposition with [11C]PiB PET,' NeuroImage, vol. 143, pp. 316-324, 2016.
[28] R. Kimura and M. Ohno, "Impairments in remote memory stabilization precede hippocampal synaptic and cognitive failures in 5XFAD Alzheimer mouse model," Neurobiology of Disease, vol. 33, no. 2, pp. 229-235, 2009.

[29] E. McGowan, J. Eriksen, and M. Hutton, "A decade of modeling Alzheimer's disease in transgenic mice," Trends in Genetics, vol. 22, no. 5, pp. 281-289, 2006.

[30] A. Willuweit, J. Velden, R. Godemann et al., "Early-onset and robust amyloid pathology in a new homozygous mouse model of Alzheimer's disease," PLoS ONE, vol. 4, no. 11, Article ID e7931, 2009.

[31] J. F. Reilly, D. Games, R. E. Rydel et al., "Amyloid deposition in the hippocampus and entorhinal cortex: Quantitative analysis of a transgenic mouse model," Proceedings of the National Acadamy of Sciences of the United States of America, vol. 100, no. 8, pp. 4837-4842, 2003.

[32] G. A. Elder, M. A. Gama Sosa, and R. de Gasperi, "Transgenic mouse models of Alzheimer's disease," Mount Sinai Journal of Medicine, vol. 77, no. 1, pp. 69-81, 2010.

[33] J. Maeda, B. Ji, T. Irie et al., "Longitudinal, quantitative assessment of amyloid, neuroinflammation, and anti-amyloid treatment in a living mouse model of Alzheimer's disease enabled by positron emission tomography," The Journal of Neuroscience, vol. 27, no. 41, pp. 10957-10968, 2007.

[34] A. M. Hall and E. D. Roberson, "Mouse models of Alzheimer's disease," Brain Research Bulletin, vol. 88, no. 1, pp. 3-12, 2012.

[35] E. O. Aboagye, P. M. Price, and T. Jones, "In vivo pharmacokinetics and pharmacodynamics in drug development using positron-emission tomography," Drug Discovery Therapy, vol. 6, no. 6, pp. 293-302, 2001. 


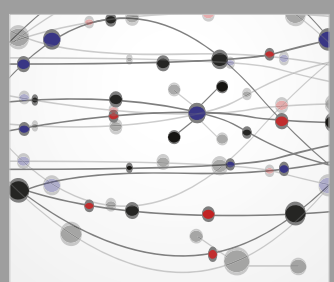

The Scientific World Journal
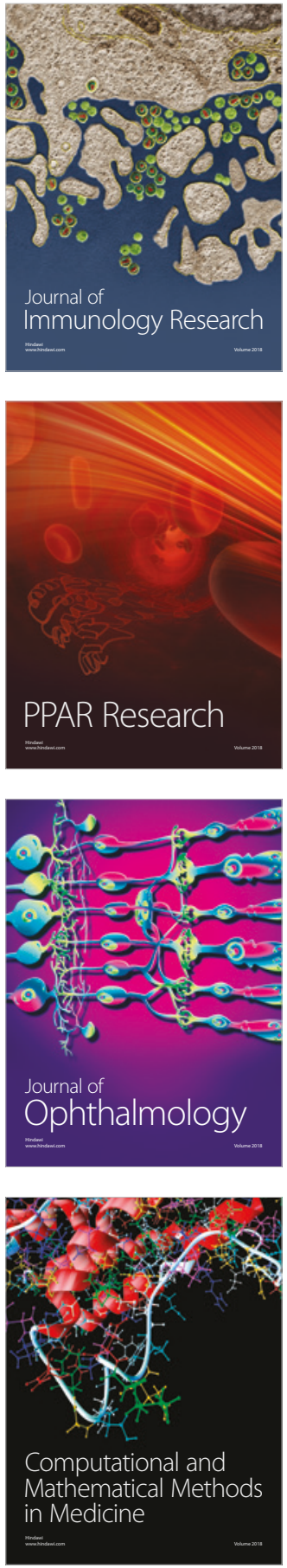

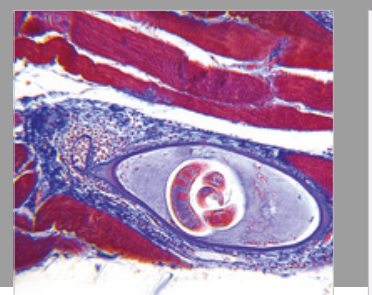

Gastroenterology Research and Practice

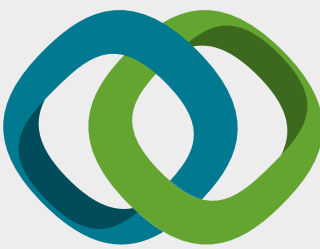

\section{Hindawi}

Submit your manuscripts at

www.hindawi.com
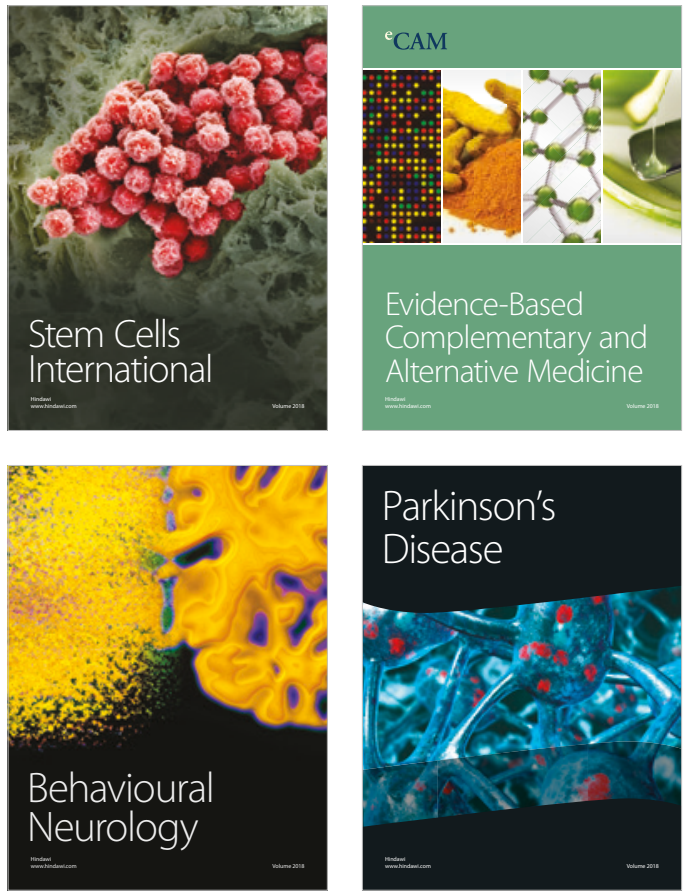

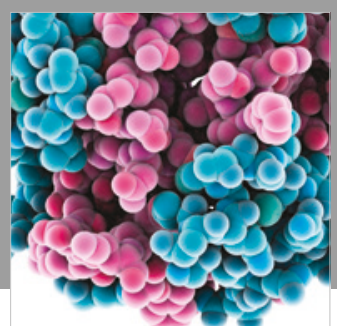

ournal of

Diabetes Research

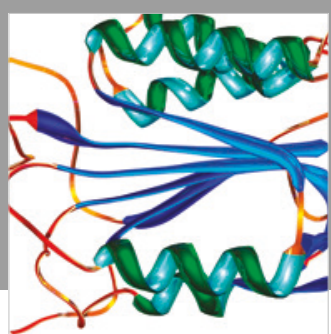

Disease Markers
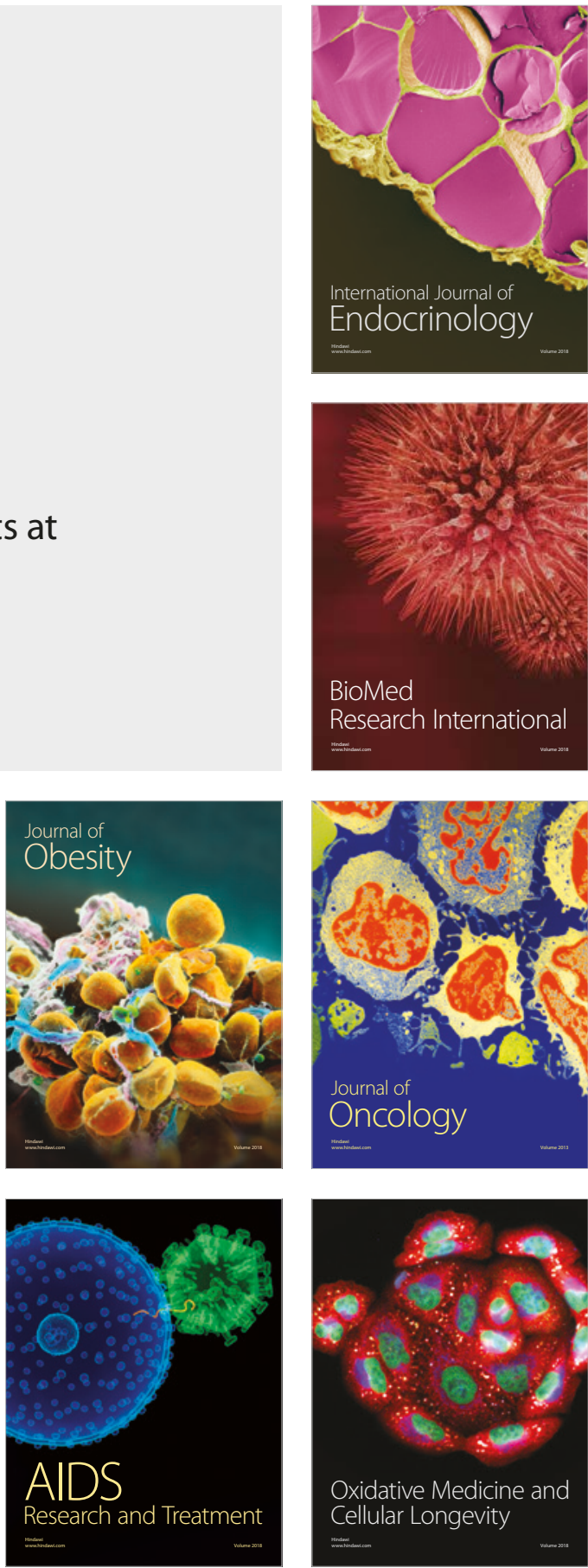